\title{
Efecto De Sobrealimentos A Base De Tres Mezclas Alimenticias (Industrial Y Natural), Sobre La Producción De Leche En Ganado Vacuno (Hostein)
}

\author{
Hilter Farley Figueroa Saavedra (Ing. Agr. Msc.) \\ Universidad / Escuela Superior Politécnica de Chimborazo Universidad / \\ Escuela Superior Politécnica de Chimborazo Extension Norte Amazónica de \\ la República del Ecuador
}

doi: 10.19044/esj.2017.v13n21p1 URL:http://dx.doi.org/10.19044/esj.2017.v13n21p1

\begin{abstract}
The main objective of this research is to evaluate the effect of three types of superfoods based on three food mixtures (artificial and natural) in the milk production of cattle (Mestiza breed). In the study, there was a reduced milk production due to the difficulties in feeding cattle. Producers use the traditional grass of low nutritional value due to the impossibility of having high cost balances as a basic problem in this preliminary work. Milk production was increased by the use of food mixtures. These mixtures are composed of different percentages of natural pastures (Gliricidia sepium, Trichanthera gigantea, Pueraria phaseoloides and Saccharum officinarum) and artificial foods (mineralized salt and Palmister obtained from palm oil). The best results in milk production occurred in treatments 5 and 6 which significantly outperformed the rest. However, the highest profitability (utility) was reached in treatment 7.
\end{abstract}

Keywords: Gmelina tree, silviculture, prunning, initial growing

\section{Resumen}

Esta investigación tuvo como objetivo principal la evaluación del efecto de tres tipos de sobrealimentos a base de tres mezclas alimenticias (artificial y natural), sobre la producción de leche de ganado vacuno (raza Mestiza). La reducida producción de leche por las dificultades en la alimentación bovina, en productores que usan pasto tradicional de bajo valor nutritivo, por la imposibilidad de contar con balanceados de elevados costos se utilizó como problema básico en este trabajo preliminar. La producción de leche se incrementó con el empleo de las mezclas alimenticias, compuestas por diferentes porcentajes de pastos naturales (Gliricidia sepium, Trichanthera gigantea, Pueraria phaseoloides y Saccharum officinarum) y 
alimentos artificiales (sal mineralizada y Palmister obtenida de la Palma aceitera). Los mejores resultados en producción de leche se produjeron en los tratamientos 5 y 6 que superaron significativamente al resto. Sin embargo la mayor rentabilidad (utilidad) se alcanzó en el tratamiento 7.

Palabras-claves: Producción de leche, pasto natural, palmister

\section{Introducción}

La ganadería en el cantón Joya de los sachas, se inició paralelo a la colonización de sus tierras, y desde esa época hasta la actualidad la alimentación del ganado se basa en el conocimiento ancestral sin el empleo de métodos modernos y como resultado la depresión en la producción de carne y leche.

En los últimos años, algunos ganaderos empíricamente se dedicaron a probar diferentes tipos de alimentación con plantas forrajeras de la zona como la Gliricidia sepium, Trichanthera gigantea, Pueraria phaseoloides y Saccharum officinarum, logrando mejorar las producciones de leche entre un 5 y $20 \%$. Las leguminosas forrajeras puras o asociadas se consideran una alternativa para mejorar la cantidad y calidad de los pastos (Urbano, 2010).

Se estima que un $25 \%$ de la producción de leche a nivel mundial se consume fundamentalmente como leche (Seow, 1997). El consumo de este producto en la zona, se eleva lo que requiere el incremento en calidad y cantidad del mismo para satisfacer las necesidades crecientes, reto que se puede cumplir con la aplicación y ayuda de nuevas e innovadoras raciones alimenticias que satisfagan los requerimientos nutritivos de cada animal y mejoren el rendimiento de la producción.

Los problemas de alimentación bovina se originan por diversas causas, entre ellas la falta de conocimiento en el empleo de forrajeras de la zona, la reducida utilización de los balanceados por sus elevados costos que obliga a los ganaderos al uso de la alimentación convencional la cual se caracteriza por el uso del pasto tradicional que posee bajo valor nutritivo.

Con estos antecedentes se propone en este trabajo la evaluación del efecto de tres tipos de sobrealimentos a base de tres mezclas alimenticias (Artificial y Natural), sobre la producción de leche de ganado vacuno.

\section{Materiales y Métodos}

El trabajo se realizó en el Cantón Joya de los Sachas pertenecientes a la Provincia de Orellana. (1 de septiembre al 30 de noviembre del 2007). La temperatura promedio en esta región oscila de 25 a $35{ }^{\circ} \mathrm{C}$, con una pluviosidad promedio anual de $3600 \mathrm{~mm}$, clima cálido húmedo, y una altitud de 400 m.s.n.m. Se seleccionó la hacienda del Sr. Cristóbal Hugo pues en 
ella se presentaron las facilidades de infraestructura diseñadas para una ganadería destinada a la producción de leche.

\section{Manejo Experimental}

El experimento se realizó durante 3 meses (Septiembre, Octubre y Noviembre) 2007. Para ello se establecieron 6 tratamientos con diferentes mezclas alimenticias y una adicional que fue el testigo donde los animales solo se alimentaron con el pasto de los potreros.

Los animales que se seleccionaron presentaron características similares tales como: 9 litros promedio de producción diaria de leche y peso que osciló entre 400 y $500 \mathrm{Kg}$, todas pertenecientes a la raza Holstein en cruce con cebuinas indubrasil y brahaman, además tenían 3 meses de gestación y una conformación lechera deseable. El ordeño se realizó dos veces al día (4.0 AM y 6.0 PM).

Los datos de la producción de leche se tomaron cada día (91 días duración del experimento) y representó la variable dependiente.

\section{Tratamientos Realizados}

Se prepararon 3 tipos de sobre alimentos a partir de las especies de leguminosas en proporciones del $80 \%$, complementando con el $20 \%$ de Saccharum officinarum, por otra parte se prepararon 3 tipos de mezclas alimenticias con balanceados comerciales PALMISTER en un 80, 90 y $100 \%$ y sal mineralizada en 20 y $10 \%$ respectivamente. La cantidad de sobrealimento y mezclas alimenticias a suministrar se realizó en proporción al peso del animal ya que se conoce que una vaca consume aproximadamente el $10 \%$ del total de su peso al día. (Tabla 1 ).

Tabla 1. Distribución de los tratamientos empleados. La unidad de medida utilizada fue $\mathrm{Kg}$

\begin{tabular}{|c|c|c|c|c|c|}
\hline Trat. & A & B & C & D & E \\
\hline T-1 & 4 & 1 & - & 4 & 1 \\
\hline T-2 & - & 1 & 4 & 4 & 1 \\
\hline T-3 & 4 & 1 & - & 4.5 & 0.5 \\
\hline T-4 & - & 1 & 4 & 4.5 & 0.5 \\
\hline T-5 & 4 & 1 & - & 3.5 & 1.5 \\
\hline T-6 & \multicolumn{7}{|c|}{ Solo Pastoreo } \\
\hline T-7 & 1 & 4 & 3.5 & 1.5 \\
\hline
\end{tabular}

A: Gliricidia sepium; B: Saccharum officinarum; C: Trichanthera gigantea; D: Pasta de Palmister; E: Sal Mineralizada

\section{Análisis Estadísticos}

Para el procesamiento estadístico se empleó el programa STATGRAPHICS plus versión 5.0. En este análisis se aplicó el método paramétrico, pues se comprobó que la distribución de la población se ajustó a 
una distribución normal, aplicando la prueba de Kolmogorov- Smirnov, además cumplió con la prueba de homogeneidad de la varianza para lo que se aplicó la prueba de Bartlett. Se utilizó el modelo lineal (ANOVA de clasificación simple); prueba de rangos múltiples de Duncan para la discriminación de las medias, con un $5 \%$ de significación.

\section{Resultados y Discusión}

En la tabla 1 se puede observar que el comportamiento de la producción de leche promedio (L) durante los tres meses evaluados se relacionó con el tratamiento.

Los mayores valores de producción de leche se obtuvieron con los tratamiento 5 y 6 los que no diferenciaron entre ellos pero si del resto. A continuación se ubican valores medios en los tratamientos 1, 2, 3, 4 .

El valor más bajo se produjo en el tratamiento 7 que se diferenció estadísticamente del resto excepto en el segundo mes que no se diferenció del tratamiento 4.

Tabla 1. Comportamiento de la producción promedio de litros de leche con relación a los tratamientos. (1 Sept/ 30 Nov. 2007)

\begin{tabular}{|c|c|c|c|}
\hline Trat. & Sept/ (L) & Oct /(L) & Nov. / (L) \\
\hline T-1 & $11,22 \mathrm{bc}$ & $11,74 \mathrm{bc}$ & $11,39 \mathrm{bc}$ \\
\hline T-2 & $11,31 \mathrm{bc}$ & $11,62 \mathrm{bc}$ & $11.52 \mathrm{bc}$ \\
\hline T-3 & $10,98 \mathrm{bc}$ & $11,66 \mathrm{bc}$ & $11,38 \mathrm{~cd}$ \\
\hline T-4 & $10,43 \mathrm{~cd}$ & $10,67 \mathrm{~cd}$ & $10,55 \mathrm{e}$ \\
\hline T-5 & $12,42 \mathrm{a}$ & $14,74 \mathrm{a}$ & $12,87 \mathrm{a}$ \\
\hline T-6 & $12,64 \mathrm{a}$ & $13,81 \mathrm{a}$ & $12,96 \mathrm{a}$ \\
\hline T-7 & $9,47 \mathrm{e}$ & $9,61 \mathrm{de}$ & $9,66 \mathrm{f}$ \\
\hline Es & 1,23 & 2,12 & 1,08 \\
\hline
\end{tabular}

Los valores representan el valor medio de tres repeticiones. Medias con letras distintas difieren para para $\mathbf{p}<\mathbf{0 , 0 5}$. Es- Error estándar. L- Litros de leche promedio.

Los resultados sugieren que la utilización de las mezclas alimenticias suplementadas con los sobrealimentos estimulan la producción de leche, a diferencia donde no se emplean pues los valores son significativamente inferiores. Se sugiere además que con el incremento en $1 \mathrm{Kg}$ de Sal Mineralizada y la disminución en $1 \mathrm{Kg}$ de la Pasta de Palmister se produce más leche.

La inclusión de las leguminosas arbóreas en las pasturas representa una tecnología viable que mejora la producción y rentabilidad en carne y leche, probablemente por el incremento de la digestibilidad, y por su contenido de proteína y algunos minerales esenciales para la nutrición de los 
bovinos (Urbano D. D., 2006). Ello sugiere que los resultados alcanzados se sustentan en el uso de las leguminosas como complemento en las mezclas.

La utilización, de niveles superiores a $2 \mathrm{~kg} / \mathrm{día} /$ cabeza del forraje fresco de Gliricidia son suficientes para aumentar la producción láctea diaria; además esta planta constituye una fuente valiosa de forraje, con un alto contenido de nutrientes, capaz de asegurar ganancias satisfactorias a costos razonables. En este trabajo se empleó $8 \mathrm{Kg}$ de Gliricidia diariamente y ello dentro de otras causas contribuyó al incremento en la producción de leche (Urbano, 2005).

Según evaluaciones, la cantidad de alimento concentrado comercial con contenido del $18 \%$ de proteína mezclado con tres tipos de pasturas reportaron que la producción de leche por vaca no fue afectada por estos factores ni por su interacción. En este estudio el uso de diferentes tipos de pasturas no afecto la producción de leche sin embargo con la reducción de pasta de Palmister se produjo una reducción de la cantidad de proteína y grasas insaturadas lo que indujo un incremento en la producción de leche. En otros resultados se informó que dietas ricas en proteínas reducen la producción en leche (Dávila, 2005).

En la tabla 3 se puede observar el comportamiento de la ganancia. Los valores más altos se presentaron en los tratamientos 5 y 6 , coincidiendo en ambos con la mayor producción de leche lo que sugiere la factibilidad del empleo de leguminosas y gramíneas, pues con su adquisición a bajos precios en el mercado y combinado con la disminución del empleo de altos volúmenes de los costosos balanceados industrializados favorece la economía de las pequeñas granjas.

Tabla 3. Comportamiento de los indicadores económicos evaluados

\begin{tabular}{|c|c|c|c|c|c|c|c|}
\hline & T-1 & $\mathbf{T - 2}$ & $\mathbf{T - 3}$ & $\mathbf{T - 4}$ & $\mathbf{T - 5}$ & T-6 & T-7 \\
\hline R (l) & 11,4 & 11,4 & 11,3 & 10,5 & 13,3 & 13,1 & 9,58 \\
\hline $\mathbf{P}(\mathbf{l})$ & 3125,8 & 3134,9 & 3059.4 & 2880,1 & 3642,7 & 3586,3 & 2615,3 \\
\hline P/V & 0,2 & 0,2 & 0,2 & 0,2 & 0,2 & 0,2 & 0,2 \\
\hline Ing & 625,1 & 626,9 & 611,8 & 576,0 & 728,5 & 717,2 & 523.0 \\
\hline G/T & 455.9 & 455.9 & 408.2 & 408.2 & 504.4 & 504.4 & 316.0 \\
\hline C/U & 0.14 & 0.14 & 0.13 & 0.14 & 0.13 & 0.14 & 0.12 \\
\hline C/P & 0,72 & 0.72 & 0.66 & 0.70 & 0.69 & 0.7 & 0.6 \\
\hline G & 169.2 & 171.0 & 203.6 & 167.8 & $\mathbf{2 2 4 . 5}$ & $\mathbf{2 1 3 . 0}$ & 207.0 \\
\hline RB & 37.1 & 37.5 & 49.8 & 41.1 & 44.5 & 42.2 & $\mathbf{6 5 . 5}$ \\
\hline
\end{tabular}

R- Rendimiento promedio (L). P- Producción total (L), P/V- Precio de venta \$, Ing- Ingreso total \$, G/T- Costo total G/T, C/U- Costo Unitario. C/P-Costo por peso invertido, G-

Ganancia. \$, RB- Rentabilidad \%.

A pesar de lograr la mayor ganancia en los tratamientos 5 y 6 la mayor rentabilidad se alcanzó en el tratamiento 7 donde solo se emplea el pasto lo 
que sugiere mayor investigación con el empleo de las mezclas alimenticias donde se logre una más alta eficiencia.

\section{Conclusiones}

El empleo de los sobre alimentos suplementado con mezclas alimenticias estimularon la producción de leche con relación al pastoreo convencional sin embargo elevan los costos lo que hacen que este último sea más rentable.

Se evidenció con la disminución de Pasta de Palmister y el incremento de Sal.

Mineralizada independiente de la combinación de pastos un incremento significativo en la producción promedio de leche.

\section{Recomendaciones}

Se recomienda evaluar, los sobre alimentos suplementado con mezclas alimenticias empleados en este ensayo pero en porcentajes diferentes.

\section{References:}

1. Dávila, C. U. (2005). Producción de leche según el tipo de leguminosa arbórea asociada y nivel de concentrado, trópico húmedo. Edición especial Tomo I.

2. Seow, C. G. (1997). Coconut milk: chemistry and technology. International Journal of Food Sciences y Technology.

3. Urbano, D. D. (2005). Leguminosas árboreas para optiizar la producción de leche y carne. Maracaibo: Astro data.

4. Urbano, D. D. (2006). Efecto de las leguminsas árboreas y la suplementación con concentrado sobre la producción de leche y cambio de peso en vacas doble propósito. Maracaibo.

5. Urbano, D. D. (2010). Efecto de la altra y frecuencia de corte sobre tres variedades de maní forrajero (Arachispintoi). Merida. 